\title{
Sensitivity Analysis of Hybrid Propulsion Transportation System for Human Mars Expeditions
}

\author{
Patrick R. Chai, ${ }^{*}$ Ryan T. Joyce ${ }^{\dagger}$ Paul D. Kessler, ${ }^{\ddagger}$ Raymond G. Merrill ${ }^{\S}$ \\ NASA Langley Research Center, Hampton, VA, 23681-2199, USA \\ Min $\mathrm{Qu}$ \\ Analytical Mechanics Associates, Inc., Hampton, VA, 23666-6413, USA
}

\begin{abstract}
The National Aeronautics and Space Administration continues to develop and refine various transportation options to successfully field a human Mars campaign. One of these transportation options is the Hybrid Transportation System which utilizes both solar electric propulsion and chemical propulsion. The Hybrid propulsion system utilizes chemical propulsion to perform high thrust maneuvers, where the delta- $\mathrm{V}$ is most optimal when applied to save time and to leverage the Oberth effect. It then utilizes solar electric propulsion to augment the chemical burns throughout the interplanetary trajectory. This eliminates the need for the development of two separate vehicles for crew and cargo missions. Previous studies considered single point designs of the architecture, with fixed payload mass and propulsion system performance parameters. As the architecture matures, it is inevitable that the payload mass and the performance of the propulsion system will change. It is desirable to understand how these changes will impact the in-space transportation system's mass and power requirements. This study presents an in-depth sensitivity analysis of the Hybrid crew transportation system to payload mass growth and solar electric propulsion performance. This analysis is used to identify the breakpoints of the current architecture and to inform future architecture and campaign design decisions.
\end{abstract}

\section{Nomenclature}

$\begin{array}{ll}\Delta M_{\mathrm{EOI}} & \text { Earth Orbit Insertion Dump Mass }(\mathrm{kg}) \\ \Delta M_{\mathrm{MOI}} & \text { Mars Orbit Insertion Dump Mass }(\mathrm{kg}) \\ \Delta M_{\mathrm{TEI}} & \text { Trans-Earth Injection Dump Mass }(\mathrm{kg}) \\ \text { BOL } & \text { Beginning of Life } \\ \text { COMPASS } & \text { Collaborative Modeling for Parametric Assessment of Space Systems } \\ \text { COPV } & \text { Composite Over-wrapped Pressure Vessel } \\ \text { EMC } & \text { Evolvable Mars Campaign } \\ \text { ISS } & \text { International Space Station } \\ M_{\mathrm{CP}} & \text { Chemical Propellant Tank Capacity }(\mathrm{kg}) \\ M_{\mathrm{HPS}} & \text { Hybrid Vehicle Dry Mass }(\mathrm{kg}) \\ M_{\mathrm{log}} & \text { Logistics \& Spares Mass (kg) } \\ M_{\mathrm{PL}} & \text { Payload Mass (kg) } \\ M_{\mathrm{Xe}} & \text { Xenon Propellant Tank Capacity }(\mathrm{kg})\end{array}$

*Aerospace Engineer, Space Mission Analysis Branch, MS 462, 1 North Dryden Street, Hampton, VA, AIAA Member.

${ }^{\dagger}$ Pathway Student, Space Mission Analysis Branch, MS 462, 1 North Dryden Street, Hampton, VA, AIAA Student Member

¥Aerospace Engineer, Space Mission Analysis Branch, MS 462, 1 North Dryden Street, Hampton, VA

$\S$ Aerospace Engineer, Space Mission Analysis Branch, MS 462, 1 North Dryden Street, Hampton, VA, AIAA Senior Member

IStaff Scientist, 21 Enterprise Parkway, Suite 300, Hampton, Virginia 


$\begin{array}{ll}\text { MALTO } & \text { Mission Analysis Low-Thruster Optimizer } \\ \text { MMH } & \text { Monomethylhydrazine } \\ \text { MSC } & \text { Mars Study Capability } \\ \text { NTO } & \text { Nitrogen Tetroxide } \\ P_{\text {BOL }} & \text { Beginning of Life Array Power (kW) } \\ P_{\mathrm{SEP}} & \text { Solar Electric Propulsion Thruster Power (kW) } \\ \text { ROSA } & \text { Roll-Out Solar Arrays } \\ \text { SARJ } & \text { Solar Alpha Rotary Joint } \\ \text { SEP } & \text { Solar Electric Propulsion } \\ T_{\mathrm{E} 2 \mathrm{M}} & \text { Earth-to-Mars Transit Time (days) } \\ T_{\text {stay }} & \text { Destination Stay Time (days) } \\ T_{\text {total }} & \text { Total Interplanetary Mission Duration (days) } \\ T_{\mathrm{M} 2 \mathrm{E}} & \text { Mars-to-Earth Transit Time (days) }\end{array}$

\section{Introduction}

B UILDING upon the success of NASA's Evolvable Mars Campaign studies, ${ }^{1,2}$ the Mars Study Capability (MSC) Team is continuing architectural trade analyses to define the capabilities and elements needed for a sustainable human presence on the surface of Mars. The development of these campaigns involves an incremental buildup of capabilities: from Earth reliant missions to expand the knowledge of operations in space, to missions in cis-lunar space for testing and certification of required technologies, and ultimately to Earth independent missions and long duration stays on the Martian surface.

Many different mission design concepts have been studied and proposed over the past three decades, ${ }^{3-5}$ and many more are currently being investigated. In the majority of these studies, chemical propulsion has been assumed for the crewed Mars missions because solar electric propulsion, despite being much more fuel efficient, produces less thrust, and is more suitable for cargo pre-deployment missions when the transit time can be much longer. The MSC Team is continuing the refinement of the new Hybrid transportation architecture in which both chemical and electric propulsion are combined in an integrated design. ${ }^{6}$ By combining chemical and solar-electric propulsion (SEP $)^{7,8}$ into a single spacecraft and applying each where it is most effective, the Hybrid architecture enables a series of Mars trajectories that are more fuel efficient than an all chemical propulsion architecture without significant increases to trip time. The Hybrid style trajectory allows the spacecraft to complete the round-trip journey to/from Mars in less than 1,100 days, which enables the reuse of the transportation system for multiple trips and eliminates the need to develop separate transportation systems for crew and cargo. For the Hybrid transportation system, a series of trajectories were designed to minimize the propulsive energy required.

NASA has been investigating the Hybrid transportation system as a potential vehicle for a sustainable human Mars campaign. Over the past few years, several papers have been published to document the progress of these studies. ${ }^{9-13}$ NASA Glenn Research Center's Collaborative Modeling for Parametric Assessment of Space Systems (COMPASS) ${ }^{14}$ Team performed a detailed design of the Hybrid Propulsion Stage for the Evolvable Mars Campaign ${ }^{1,2}$ (EMC). This effort resulted in a single baseline vehicle which was utilized for the initial feasibility study outlined in previous papers. ${ }^{9,10}$ This baseline vehicle has been continuously refined since the last publication. The major modifications include upgrading to higher specific impulse chemical engines, ${ }^{15}$ increased solar array beginning of life (BOL) power, and increased tank capacity to accommodate more propellant. These modifications are part of the effort to increase the performance of the Hybrid vehicle to accommodate increases in payload mass and other changing requirements. The updated vehicle's design summary and mass breakdown are shown in Figure 1. Although the modifications were relatively minor, the refinement activities highlight the sensitivity of the vehicle's performance to changing requirements. To be able to design a robust transportation system that can be utilized across an entire campaign of missions, these sensitivities must be fully understood.

This paper presents an in-depth sensitivity analysis of the Hybrid crew transportation architecture to payload mass growth. Previous studies considered single point designs of the architecture, with fixed payload mass and propulsion system performance parameters. As the architecture matures, it is inevitable that the payload mass and the performance of the propulsion system will change. It is desirable to understand how 

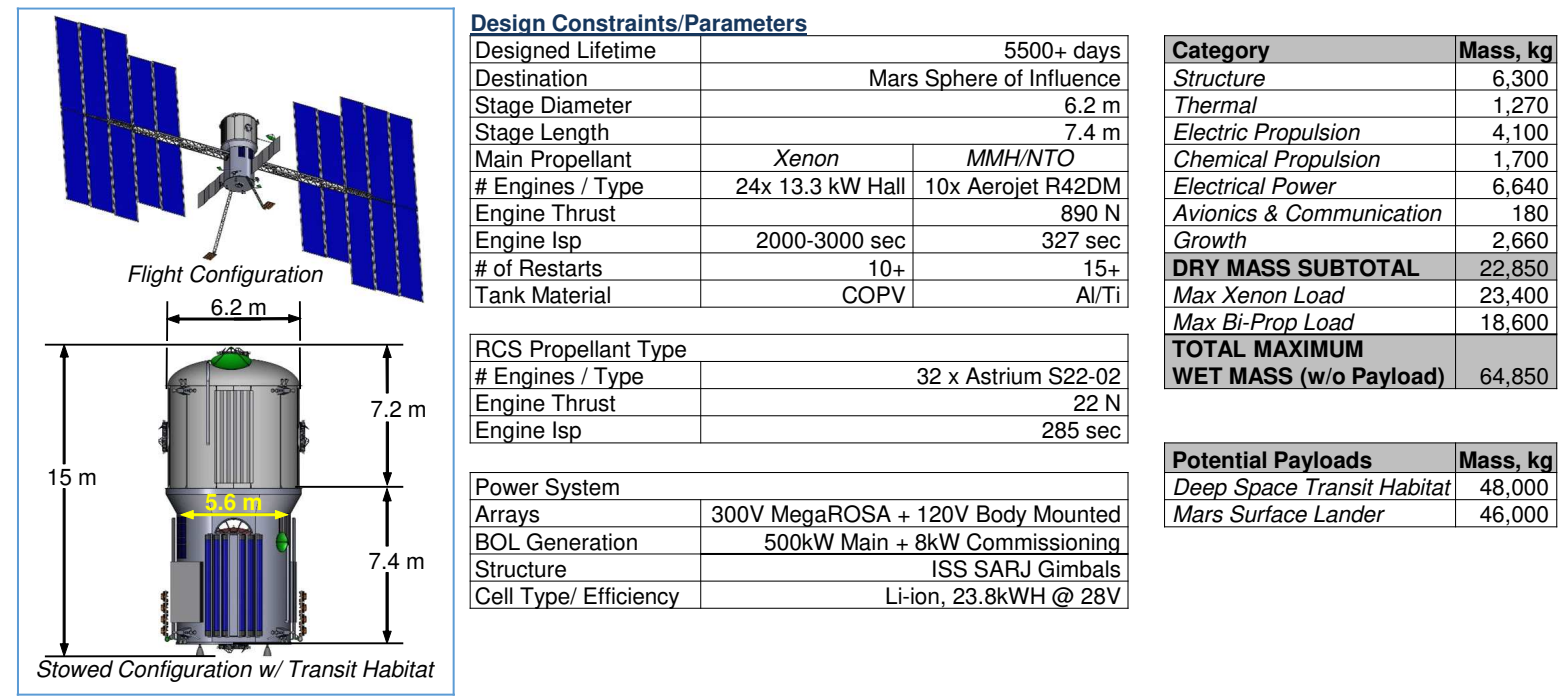

\begin{tabular}{|l|r|}
\hline Potential Payloads & Mass, kg \\
\hline Deep Space Transit Habitat & 48,000 \\
\hline Mars Surface Lander & 46,000 \\
\hline
\end{tabular}

Figure 1: Hybrid Propulsion System Design Summary

these changes will impact the in-space transportation system's mass and power requirements. Previously published papers have shown that the currently designed Hybrid transportation system can only accommodate very minor growth of the habitat mass. ${ }^{9,10}$ Additional mass growth will require more propellant than the current capacity of the tanks, additional array area and mass, or increased electric propulsion thruster power. This analysis could be used to identify the breakpoints of the current architecture and to inform future architecture and campaign design decisions.

\section{Trajectory Optimization and Sizing}

In order to understand the sensitivity of the transportation system to architecture level requirements, a fully integrated trajectory optimization and spacecraft sizing tool was developed. The tool integrates NASA Jet Propulsion Laboratory's Mission Analysis Low-Thrust Optimizer ${ }^{16}$ (MALTO) with a vehicle sizing routine to provide vehicle closure and ensure the vehicle's performance meets the specified requirements. Detailed descriptions of potential hybrid trajectories have been published in previous studies. ${ }^{11,12}$ The analysis tool utilizes the trajectory information provided by MALTO to size the low-thrust propellant tanks based on parametric scaling from the baseline vehicle and computes the propellant required from high thrust maneuvers to size the chemical propellant tanks. Due to the circular nature of the sizing routine, a fixed point iteration process was implemented to achieve vehicle closure.

The parametric model for the Hybrid vehicle was developed to approximate the vehicle's mass as function of five major inputs: SEP thruster power, BOL array power, xenon propellant capacity, and chemical propellant capacity, and payload mass. The parametric model utilizes the detailed mass breakdown from the COMPASS design and scales each component to account for the changing vehicle requirements. For example, the vehicle's integrated structure is scaled based on the total vehicle wet mass, as the structure must be able to withstand both launch loads on Earth and thrust loads in space. The resulting parametric model takes the form of a multivariate linear regression with five independent variables shown in Equation 1 , where $M_{\mathrm{HPS}}$ is the dry mass of the Hybrid propulsion system in kilograms, $P_{\mathrm{SEP}}$ is the power of the SEP thrusters in kilowatts, $P_{\mathrm{BOL}}$ is the BOL power that the solar arrays provide at $1 \mathrm{AU}$ in kilowatts, $M_{\mathrm{Xe}}$ is the xenon propellant tank capacity in kilograms, $M_{C P}$ is the chemical propellant tank capacity in kilograms, and $M_{\mathrm{PL}}$ is the payload mass for the transportation system.

$$
M_{\mathrm{HPS}}=2672+12.465 P_{\mathrm{SEP}}+15.941 P_{\mathrm{BOL}}+0.0586 M_{\mathrm{Xe}}+0.272 M_{\mathrm{CP}}+0.0232 M_{\mathrm{PL}}
$$

The payload mass in this analysis includes both the dry mass of the deep space transit habitat and the logistics/spare mass required to support the roughly 1,100 day mission. The logistics and spares masses are 
not fixed for the different missions and sensitivity runs. Each case has slightly different transit and destination duration, which impacts the logistics and spares masses; thus for each run, the logistics and spares masses are computed with a separate parametric equation. Additionally, prior to each major chemical maneuver, a portion of the waste is disposed to increase the propulsion system performance; these waste disposals are also a function of transit and stay time. The parametric models for the logistics and spares masses were derived from data generated for a previous trash disposal study. ${ }^{17}$ The logistics mass and the associated waste disposal mass are shown in Equations 2-5. $M_{\log }$ is the total logistics and spares mass required prior to Earth departure in kilograms. $\Delta M_{\mathrm{MOI}}, \Delta M_{\mathrm{TEI}}, \Delta M_{\mathrm{EOI}}$, are the waste disposal masses prior to the Mars Orbit Insertion, Trans Earth Injection, and Earth Orbit Insertion maneuvers respectively in kilograms. $T_{\text {total }}$ is the total duration for the crew from Earth departure to Earth return in days, $T_{\mathrm{E} 2 \mathrm{M}}$ is the Earth to Mars duration in days, $T_{\mathrm{M} 2 \mathrm{E}}$ is the duration from Mars to Earth in days, and $T_{\text {stay }}$ is the destination stay time duration in days.

$$
\begin{aligned}
M_{\text {log }} & =19.62 * T_{\text {total }}+1788 \\
\Delta M_{\mathrm{MOI}} & =8.006 * T_{\mathrm{E} 2 \mathrm{M}}-1.667 \\
\Delta M_{\mathrm{TEI}} & =2.689 * T_{\mathrm{E} 2 \mathrm{M}}+12.778 * T_{\text {stay }}-25.414 \\
\Delta M_{\mathrm{EOI}} & =8.006 * T_{\mathrm{M} 2 \mathrm{E}}-2.048
\end{aligned}
$$

To achieve vehicle closure, the analysis tool first provides an initial guess of the Earth departure mass of the entire transportation system. With the initial mass guess, the tool utilizes MALTO ${ }^{16}$ to compute the low thrust portion of the trajectory and outputs the planetary departure and arrival conditions, which allows for computation of the departure and arrival maneuvers necessary to achieve the appropriate hyperbolic excess velocity. Additional maneuvers such as the orbital maintenance and orbit reorientation are computed to determine the total amount of chemical and electric propulsion propellant required to satisfy the trajectory. The propellant requirements are utilized to size the transportation vehicle, which is iterated against the final mass of the trajectory optimization to ensure the closure. This process is repeated for each of the cases that are of interest.

\begin{tabular}{|l|c|}
\hline Mission Years & 2033, 2037, 2041, 2045 \\
\hline SEP Thruster Power & $318 \mathrm{~kW}$ \\
\hline BOL Array Power & $500 \mathrm{~kW}$ \\
\hline Payload & $48 \mathrm{t}$ \\
\hline
\end{tabular}

Table 2: Baseline Mission Parameters

To understand the sensitivity of the transportation system to the design variables, baseline missions are defined to provide an anchor point for the comparisons. The transportation system baseline mission parameters are show in Table 2 and are taken from the current MSC study team's ground rules and assumptions. The MSC team currently is investigating crewed missions to Mars sphere of influence beginning in 2033. The typical mission cadence for a Mars campaign fields a crewed mission every other Earth-Mars opportunity. Thus, for this analysis, the baseline missions are in 2033, 2037, 2041, and 2045. The SEP thruster power remains the same from previous EMC studies ${ }^{1,2}$ while the BOL array power has been increased to $500 \mathrm{~kW}$ due to the increase in the payload mass. The current payload mass includes a $24 \mathrm{t}$ dry deep space transit habitation module as well as $24 \mathrm{t}$ of logistics and spares.

The baseline mission system mass summary is shown in Figure 2. Across the the four opportunities of interest, the variation of the transportation system can be significant due to the varying planetary orientation across the Earth-Mars synodic period. The Hybrid dry mass shows variation of about one ton, but the propellant mass varies from 50t to $56 \mathrm{t}$. From the baseline mission results, the 2033 opportunity requires the least amount of propellant, while the 2045 opportunity requires the most. Examining the baseline mission summary shows the significant variation to the propulsive performance requirement across the different opportunities. A few observations can be made from these baseline missions. First, the masses are higher than in previously published studies ${ }^{9-11}$ due to increases in the payload mass as well as additional trajectory refinements that resulted in increases in the delta- $\mathrm{V}$ requirements for the maneuvers that are outside of the trajectory optimization. These maneuvers will likely be further refined as the deign of the system matures. 


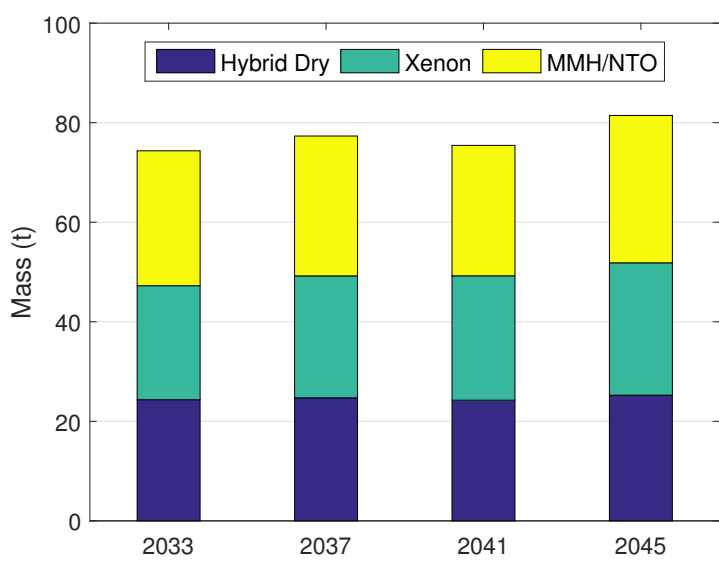

\begin{tabular}{|l|ccc|c|}
\hline & Hybrid & & MMH/ & Hybrid \\
& Dry & Xenon & NTO & Wet \\
\hline 2033 & $24.4 \mathrm{t}$ & $22.9 \mathrm{t}$ & $27.1 \mathrm{t}$ & $74.4 \mathrm{t}$ \\
2037 & $24.7 \mathrm{t}$ & $24.5 \mathrm{t}$ & $28.1 \mathrm{t}$ & $77.3 \mathrm{t}$ \\
2041 & $24.2 \mathrm{t}$ & $25.0 \mathrm{t}$ & $26.2 \mathrm{t}$ & $75.4 \mathrm{t}$ \\
2045 & $25.3 \mathrm{t}$ & $26.6 \mathrm{t}$ & $29.6 \mathrm{t}$ & $81.5 \mathrm{t}$ \\
\hline
\end{tabular}

Figure 2: Baseline Mission Masses Across Multiple Opportunities with 48t Payload, 318kW SEP Thruster Power, and 500kW BOL Array Power

Second, the amount of chemical propellant can be significantly more than the amount of electric propulsion propellant. The low thrust portion of the trajectory is relatively inflexible with fixed power, as the thruster power defines the propellant throughput and the array power defines how much the thruster can be utilized. If the system is under-powered or the payload grows, the high-thrust chemical system must make up the difference with additional maneuvers at planetary departures and arrivals. Both of these observations will be amplified in the sensitivity analysis.

\section{Sensitivity Results \& Discussions}

\begin{tabular}{|l|c|}
\hline Mission Years & 2033, 2037, 2041, 2045 \\
\hline SEP Thruster Power & $265-504 \mathrm{~kW}$ \\
\hline BOL Array Power & $400-600 \mathrm{~kW}$ \\
\hline Payload & $40-64 \mathrm{t}$ \\
\hline
\end{tabular}

Table 3: Sensitivity Analysis Variation Parameters

For the sensitivity analysis, the variations of the input parameters are summarized in Table 3. For each of the mission opportunities, the SEP thruster power, BOL array power, and the payload were varied to determine the HPS mass required to field a single round-trip mission. The SEP thruster power was varied between $265 \mathrm{~kW}$ to $505 \mathrm{~kW}$ by changing the number of thrusters that was available. The HPS SEP thrusters are $13.3 \mathrm{~kW}$ class thrusters, so the variation was the results of a sweep between 20 and 38 thrusters with 24 thrusters being the nominal case. The BOL array power was varied between $350 \mathrm{~kW}$ and $600 \mathrm{~kW}$ with $50 \mathrm{~kW}$ increments, with $500 \mathrm{~kW}$ as the baseline power. Cases in which the SEP thruster power was greater than the BOL array power were considered to be non-practical and thus were eliminated. Finally, the payload was varied between 40 and $64 \mathrm{t}$ by varying the deep space habitation dry mass from 16t to $40 \mathrm{t}$. The logistics and spares mass was then computed from the parametric model described in Equations 2-5 which adds approximately $24 \mathrm{t}$ to the dry mass, resulting in a total payload variation between $40 \mathrm{t}$ and $64 \mathrm{t}$ at Earth departure. A total of 750 data points were generated across the four mission opportunities with varying parameters to capture a small portion of this complex design space. The wet mass of the HPS, comprised of the vehicle dry mass and the electric propulsion and chemical propulsion propellant mass, is used as the primary metric.

Figure 3 shows the first sensitivity analysis of interest. The plot shows the HPS wet mass as a function of the SEP thruster power with fixed 500kW BOL array and 48t payload across the four mission opportunities. From the plot, one can see clearly that each mission opportunity has its own performance behavior that is independent of each other. Across the opportunities, the optimal trajectories utilize the SEP and chemical 


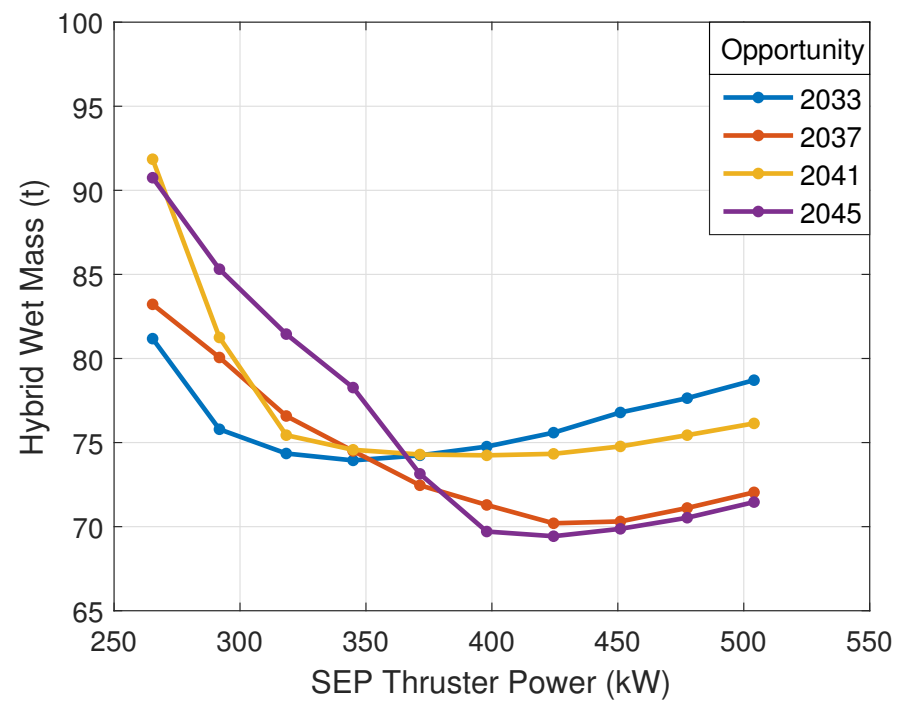

Figure 3: Hybrid Wet Mass Sensitivity to SEP Thruster Power with Fixed 500kW BOL Array Power and 48t Payload for the 2033, 2037, 2041, and 2045 Mission Opportunities

system differently depending on the planetary alignment and the power level available to the transportation system. As the SEP thruster power decreases, the system relies increasingly more on the chemical propulsion system, which is less efficient than the SEP system, to make up for the lack of SEP thrusters. This is evident in the figure, as the HPS wet mass grows dramatically when the SEP thruster power decreases from the opportunity-specific optimum value. This sensitivity highlights one of the challenges of designing a single spacecraft to be reusable for multiple missions to Mars. The figure shows the mass optimal SEP thruster power for the 2033 mission opportunity given the fixed variable is roughly $350 \mathrm{~kW}$, while the 2037 , 2041, and 2045 mission opportunities have mass optimal thruster power of $425 \mathrm{~kW}, 400 \mathrm{~kW}$, and $425 \mathrm{~kW}$ respectively. These variations make it difficult to design a single vehicle that performs the mission across opportunities as the vehicle will be flying non-optimal trajectories. Further, the current SEP thruster power is $318 \mathrm{~kW}$, which is on the steep portion of the mass curve. This means that any reduction of the SEP thruster power can have a dramatic impact on the performance of the Hybrid propulsion system. A more robust design would see the SEP thruster power increase to the $400 \mathrm{~kW}$ to $450 \mathrm{~kW}$ range to guard against potential decrease in thruster performance for the $48 \mathrm{t}$ payload case.

Figures $4 \mathrm{a}$ through $4 \mathrm{~d}$ show plots of the Hybrid wet mass as a function of the SEP thruster power and BOL array power with a fixed 48t payload for the four mission opportunities. For each plot, each of the lines represent fixed BOL array power level as the thruster power is varied. For some opportunities, the lower BOL array power line is not available due to the difficulty in the vehicle achieving performance closure. For all four opportunities, the trend observed in Figure 3 remains: the Hybrid wet mass increases dramatically as the thruster power is decreased. However, the slope of the trend and the inflection point of the curve is different from opportunity to opportunity.

For the 2033 mission opportunity, shown in Figure 4a, the HPS wet mass is insensitive to the SEP thruster power above $350 \mathrm{~kW}$. However, as the SEP thruster power drops below $350 \mathrm{~kW}$, the Hybrid wet mass growth quickly becomes exponential. For the 2033 mission opportunity, the optimal BOL array power from a mass perspective is $600 \mathrm{~kW}$, which is the maximum BOL array power considered in this study. The $550 \mathrm{~kW}$ BOL array power represents a mass increase of roughly $1 \mathrm{t}$ compared to the $600 \mathrm{~kW}$ BOL case, though for SEP thruster power less than $300 \mathrm{~kW}$, the $500 \mathrm{~kW}$ BOL array power results in a lower Hybrid wet mass. In contrast to the other mission opportunities, the 2033 mission opportunity is highly sensitive to the BOL array power. Examining Figures $4 \mathrm{~b}, 4 \mathrm{c}$, and $4 \mathrm{~d}$, the relative difference between the different BOL array power curves is significantly less than the 2033 mission in Figure 4a. This is particularly clear in the 2041 mission opportunity, shown in Figure 4c, as all of the BOL array curves are almost indistinguishable from one another.

The 2033 and the 2041 mission opportunities exhibit similar sensitivity to the SEP thruster power. Both 


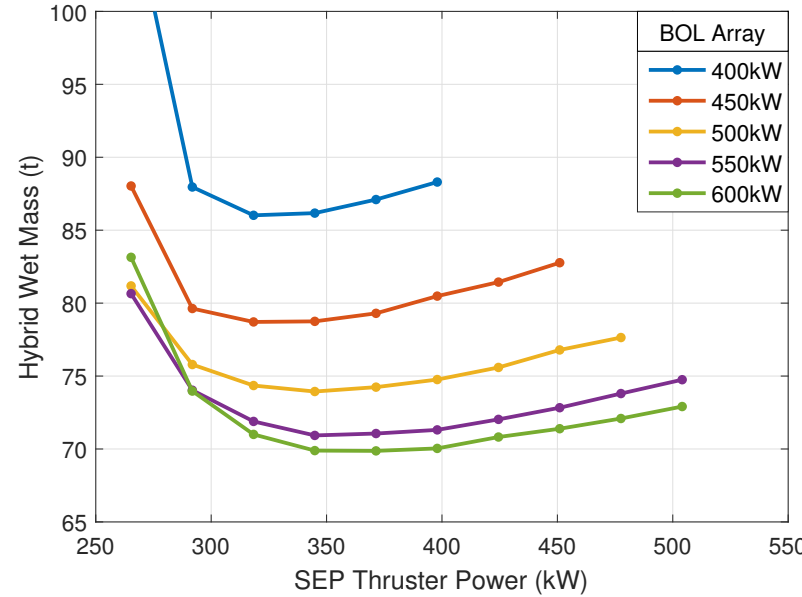

(a) 2033 Mission Opportunity

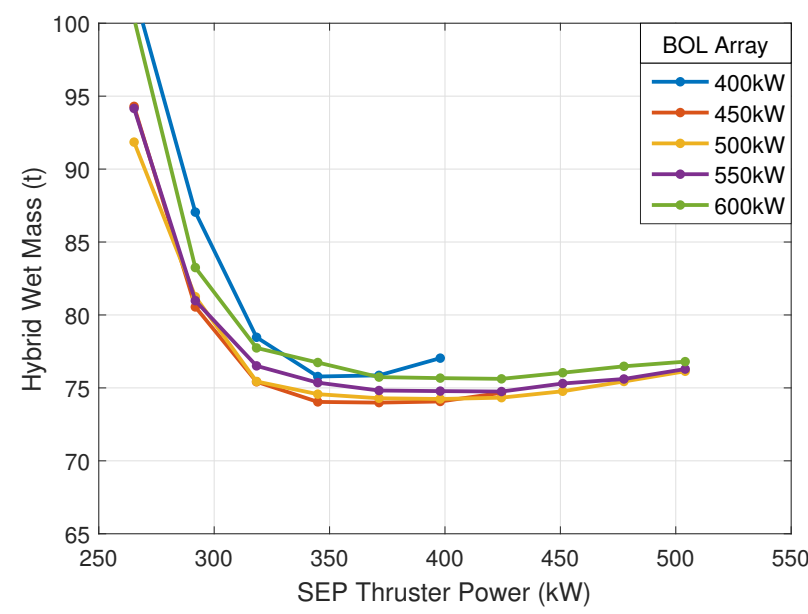

(c) 2041 Mission Opportunity

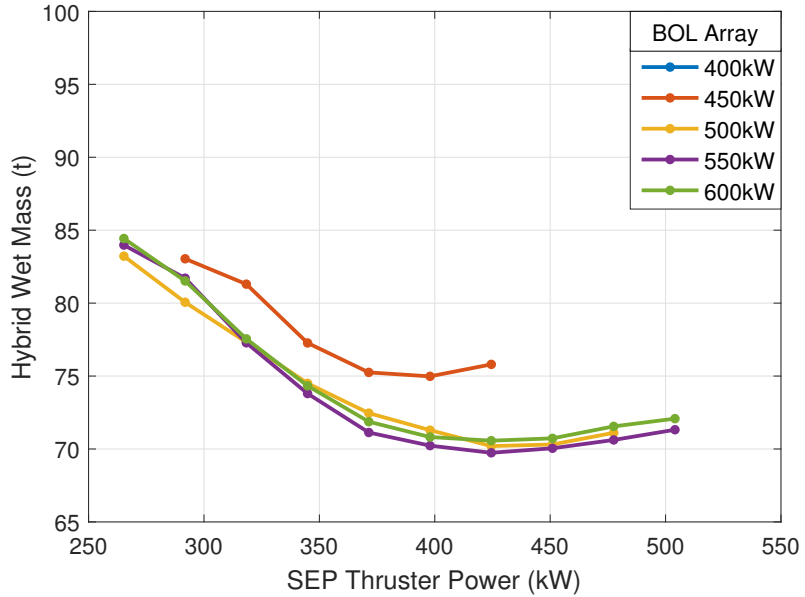

(b) 2037 Mission Opportunity

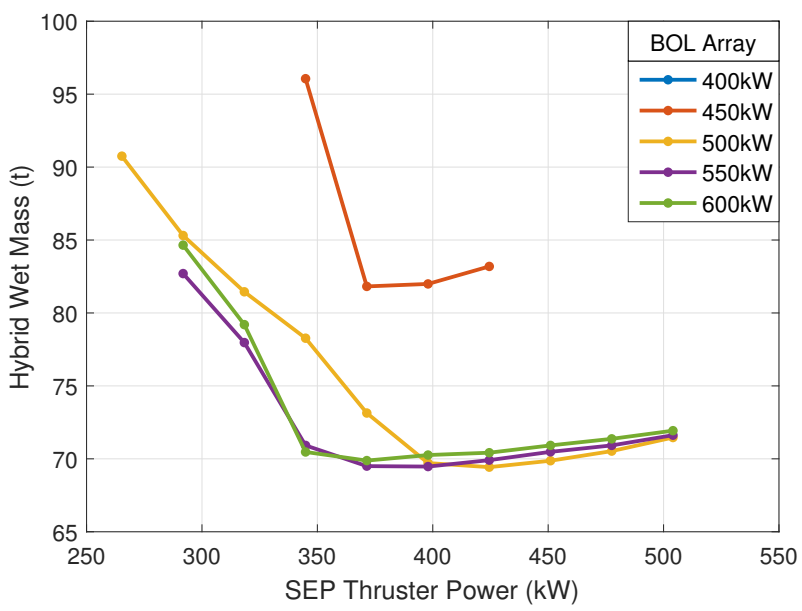

(d) 2045 Mission Opportunity

Figure 4: Hybrid Wet Mass Sensitivity to SEP Thruster Power and BOL Array Power with Fixed 48t Payload Across the Four Mission Opportunities

of these opportunities have mass curves of similar shape with similar inflection points. For SEP thruster power greater than $350 \mathrm{~kW}$, the Hybrid wet mass is relatively insensitive to additional SEP thruster power. However, reductions in the SEP thruster power will result in exponential increases in the Hybrid wet mass. For the 2037 opportunity, the mass sensitivity to the SEP thruster power is not as significant as the 2033 and 2041 opportunities as evident by the relatively flat curves show in Figure 4b. The mass growth due to the lack of SEP thruster power still exists for the 2037 mission opportunity; however, in the design space defined in this study the mass growth does not reach the exponential portion of the curve for the 2037 mission opportunity. From examining the results of the other mission opportunities, it can be inferred that as SEP thruster power is reduced further, the mass growth will eventually become exponential in nature. The lower the SEP thruster power, the more the system relies on the chemical propulsion system to achieve the desired orbital energy for the trajectory, and the exponential nature of the rocket equation governs the mass growth in this region of the design space.

The 2037 mission opportunity also has no 400kW BOL array power curve. Even though the opportunity is relatively insensitive to the BOL array power, the $400 \mathrm{~kW}$ power case is infeasible for this opportunity as well as the 2045 mission opportunity. For both of these mission opportunities, the sensitivity to the array power is minimal when the power is above $500 \mathrm{~kW}$. However, as the BOL array power dips below 500kW, the design space becomes significantly more sensitive and restrictive. As seen in the $450 \mathrm{~kW}$ curve for the 2045 mission opportunity, the area in which the mass is insensitive to thruster power change is very small. 
These curves shows a glimpse of the total design space for the Hybrid transportation system and how the trajectory optimization and system sizing are impacted by the region in which the SEP thruster power and BOL array power operates. This finding highlights the challenge in selecting a vehicle concept that is robust across multiple mission opportunities.
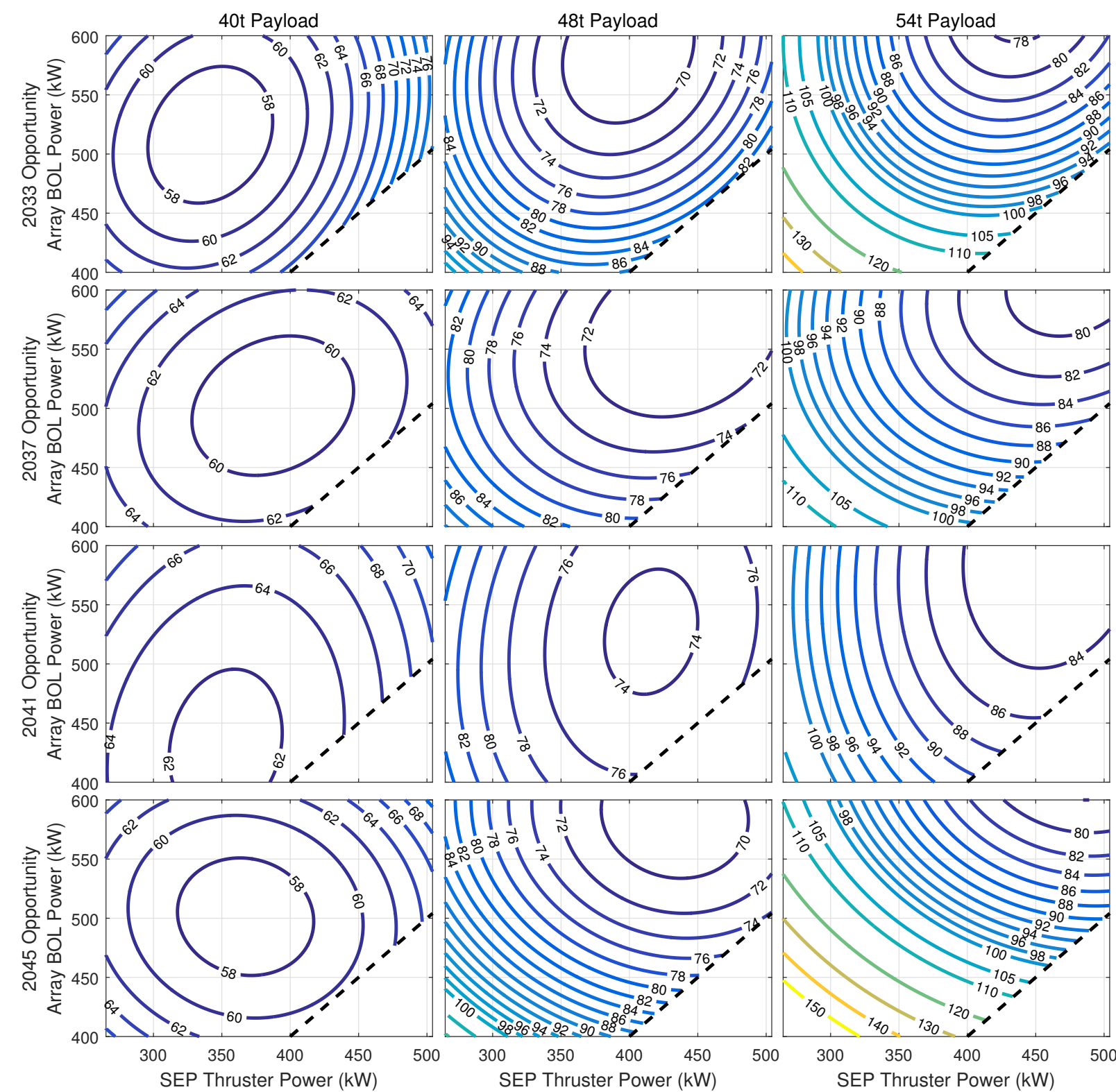

Figure 5: Hybrid Wet Mass Contours (t) as Functions of SEP Thruster Power and BOL Array Power for the Four Mission Opportunities and Fixed 40t, 48t, and 54t Payload

The discussions and analyses presented so far have focused on the variation of the SEP thruster power and the BOL array power with a fixed payload. One of the major risks to the in-space transportation system is the growth of the payload mass. As payload mass grows, the demands on the propulsion system can increase dramatically. The sensitivity of the transportation to the other design variables can be dramatically different as the payload mass changes. Figure 5 shows the constant contour plots for the Hybrid wet mass as a function of the SEP thruster power and the BOL array power for the four mission opportunities and fixed 40, 48, and 54t payloads. The contour plots provide a more comprehensive look at the Hybrid transportation system design space by showing the vehicle mass sensitivity to the two power variables at the same time. The contour lines show fixed $2 \mathrm{t}$ increment from $58 \mathrm{t}$ to $100 \mathrm{t}$, then shows the mass growing by $10 \mathrm{t}$ increments 
beyond 105t. Thus, up to 100t, the distance between the contour lines shows the sensitivity of the hybrid wet mass. The closer the contour lines, the more sensitive the hybrid wet mass is to the changing variables.

The middle column of plots represents the nominal payload mass for the transportation system, which is the same as the data shown in Figure 4. From the middle column of the plots, it is immediately clear that given a 48t payload, the transportation system mass is less sensitive to power level changes when the BOL array power is above $500 \mathrm{~kW}$ and the SEP thruster power is above $400 \mathrm{~kW}$. The other two columns of plots show the change to the behavior of the mass sensitivity for different payload mass. The 40t payload case in the first column, corresponding to a habitat dry mass of 16t, shows the Hybrid wet mass is relatively insensitive to the power levels. In fact, the variation of both the SEP thruster power and the BOL array power results in Hybrid wet mass variation between $58 \mathrm{t}$ and $70 \mathrm{t}$. Compared to the $48 \mathrm{t}$ payload case, in which the Hybrid wet mass varied between $70 \mathrm{t}$ and more than 95t. This shows how much more performance margin the Hybrid transportation system has with a lower payload mass. Indeed, when the Hybrid transportation system was first conceived, the payload mass was approximately $40 t,{ }^{9}$ which partially justified the choice of the power level at the time $(450 \mathrm{~kW}$ BOL array and $318 \mathrm{~kW}$ SEP thruster). However, as this analysis shows, the $8 \mathrm{t}$ increase in the payload mass results in the Hybrid system being much more sensitive to the variation in the power levels.

Additional increases to the payload mass further increase the sensitivity of the Hybrid wet mass to the two power variables. This is shown in the right most column of plots in Figure 5, which shows the Hybrid

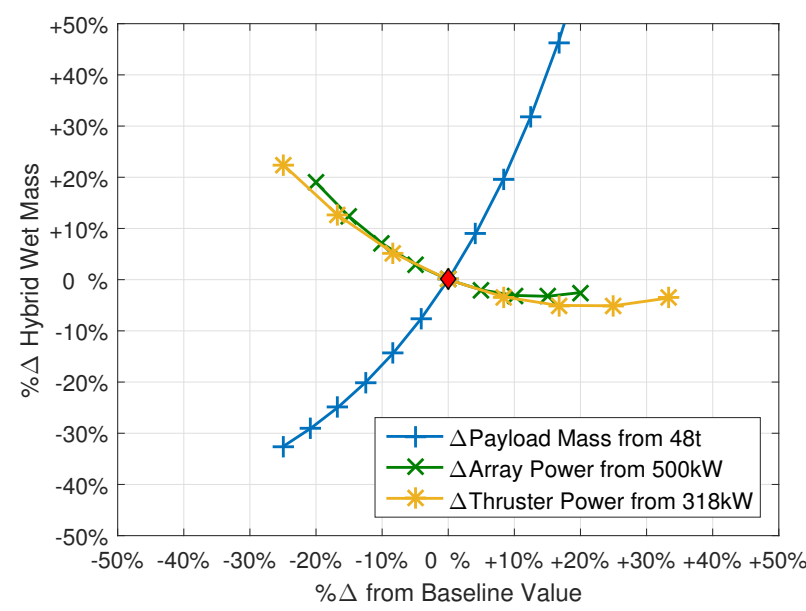

(a) 2033 Mission Opportunity

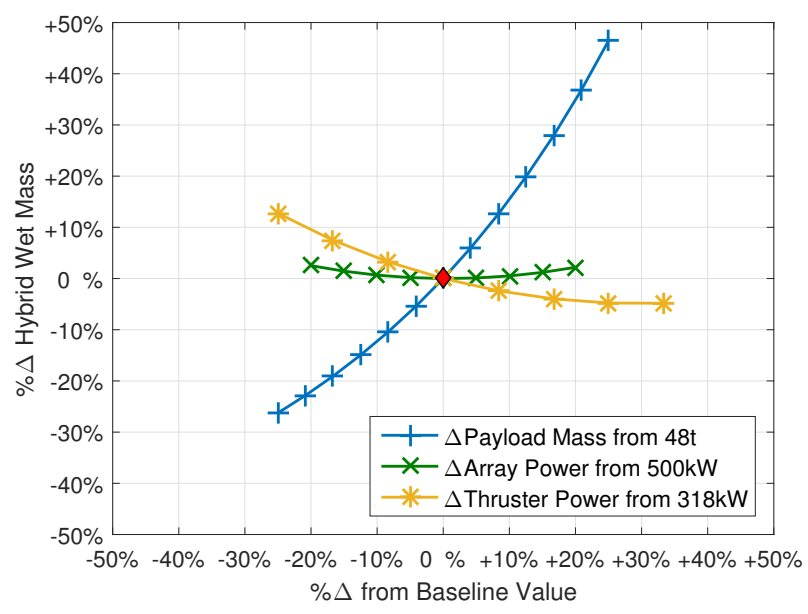

(c) 2041 Mission Opportunity

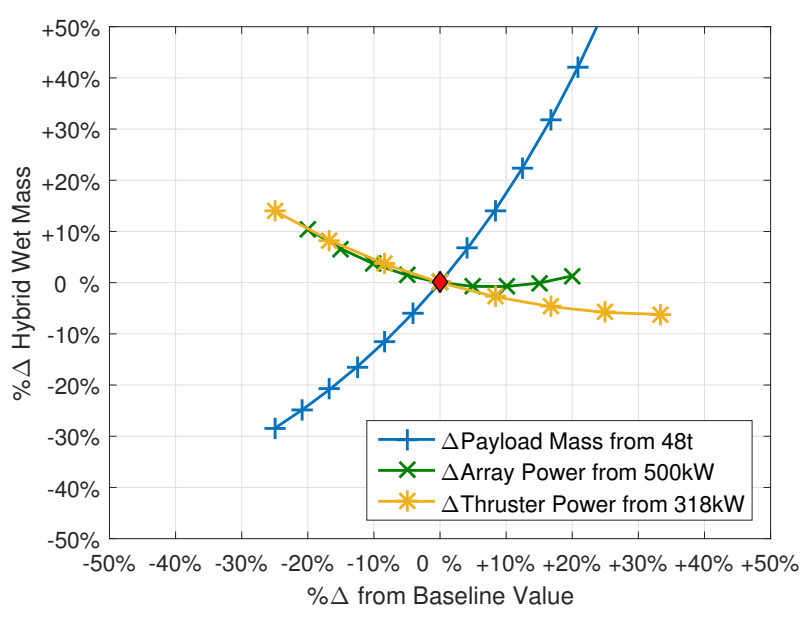

(b) 2037 Mission Opportunity

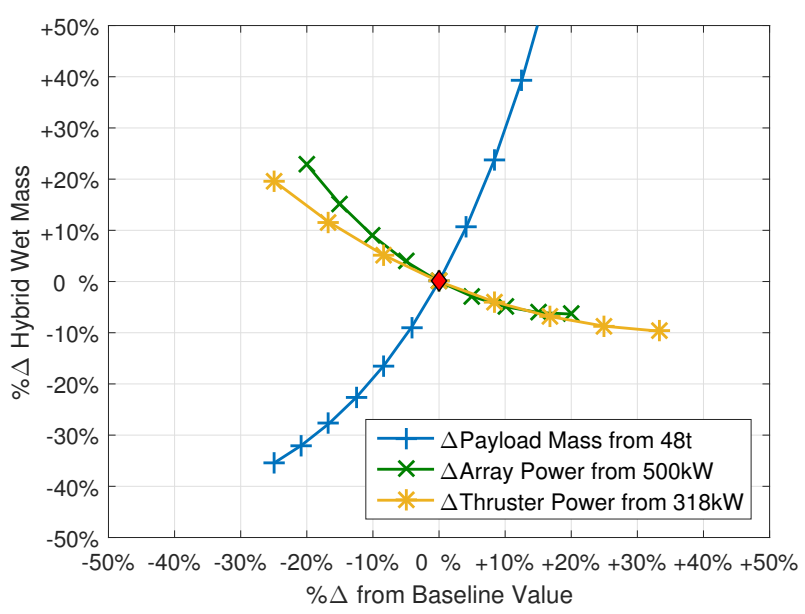

(d) 2045 Mission Opportunity

Figure 6: Sensitivity of the Hybrid Wet Mass as a Function of the Payload Mass, BOL Array Power, and SEP Thruster Power normalized to the Baseline Value 
mass contour for the 54t payload (30t dry habitat) case. As the plots show, the current power level is in an area in which the Hybrid wet mass is very sensitive to power changes, and the range of the wet mass change is significantly higher than the lower payload cases. The robust part of the design space is so far to the top right of the plot that it is outside the scope of the current study. Although some of the opportunities are slightly more forgiving when it comes to payload mass increase, the general trend of increased sensitivity for higher payload mass holds.

Another interesting trend that can be observed from the contour plots shown in Figure 5 is how the different opportunities result in significantly different sensitivity to the two power variables. This can be seen from examining the shape of the "grain" of the contour plots. For the 2033 opportunities, it can be seen that the Hybrid wet mass is equally sensitive to both the BOL array power and the SEP thruster power, as moving in either direction will cause significant changes to the Hybrid wet mass. This same trend is observed in the 2037 and 2045 opportunities. However, the 2041 opportunity does not exhibit the same trend. The 2041 opportunity is relatively insensitive to the BOL array power, especially compared to the sensitivity to the SEP thruster power. At any given SEP thruster power, changes to the BOL array power results in little to no change to the HPS wet mass. This finding further highlights the importance of evaluating all of the mission opportunities when designing the Hybrid transportation system.

The varying sensitivity across the different opportunities can be more clearly seen in the plots shown in Figure 6. In these plots, the y-axis represents the percent change of Hybrid wet mass from the baseline mass and the $\mathrm{x}$-axis represents the percent change of the three different variables from their respective baseline values. The percentage change allows for all three variables to be plotted on the same axis. As the four plots show, the payload mass has a dramatic impact on the Hybrid wet mass. The percentage change to the Hybrid wet mass due to the payload mass shows minor variations across the four mission opportunities. A $20 \%$ decrease in payload mass at the baseline power results in a 20-30\% decrease in the Hybrid wet mass, and a $20 \%$ increase in the payload mass results in 30-60\% increase in the Hybrid wet mass. The plots show that the current power levels are at the edge of the performance boundary for the Hybrid vehicle from a payload mass standpoint, as increases to the payload mass significantly increase the Hybrid wet mass.

The Hybrid wet mass sensitivity to the BOL array power is more variable across the four mission opportunities. The plots show that in the 2037 and 2041 opportunities the Hybrid wet mass is relatively insensitive to the BOL array power, while the 2033 and 2045 opportunities are more sensitive, especially as the power decreases. For the 2037 and 2041 opportunities, a plus or minus $20 \%$ change to the BOL array power results in less than 10\% change in the Hybrid wet mass. The 2041 opportunity is especially insensitive to the BOL array power, as the Hybrid wet mass shows less than $5 \%$ change for a $20 \%$ change in the array power. In contrast, the 2033 and 2045 opportunities show a 20-30\% increase in Hybrid wet mass for a $20 \%$ decrease in the BOL array power. Again, the variations across the different mission opportunities is the result of the variation of the planetary alignments across the Earth-Mars synodic period. As Earth and Mars orbit around the Sun, the distance and the relative velocity between the two planet change, and thus a spacecraft traveling from one to the other will require different orbital energy. The Hybrid vehicle is unique in that it can utilize both the SEP and chemical system to change the spacecraft's orbital energy. Depending on the mission opportunity and SEP power levels, the balance between the two propulsion system can be drastically different.

For the SEP thruster power, all four opportunities show similar Hybrid wet mass sensitivity at the current payload mass. The 2033 and 2045 mission opportunities are slightly more sensitive to the SEP thruster power than the 2037 and 2041 mission opportunities, however, the trend of the sensitivity is the same. A 20\% decrease in the SEP thruster power results in an increase in the Hybrid wet mass by 10$20 \%$. Increase to the SEP thruster power can reduce the Hybrid wet mass, however, as the 2033 mission opportunity shows, too much increase in the SEP thruster power will results in increase to the Hybrid wet mass from the mass optimal solution. It is important to note that the SEP thruster power will likely never change by itself. It is more likely that changes to the SEP thruster power will go in tandem with changes to the BOL array power, which can have dramatic impact to the overall HPS mass as discussed previously.

Finally, plots in Figure 7 show how the Hybrid wet mass sensitivity to the two power variables changes for different payload masses across the four mission opportunities. For each of the plots, the BOL array power and the SEP thruster powers are varied from their respective nominal value for three different payload masses. The y-axis shows the percent change of the Hybrid wet mass from the baseline values from Table 2. The 48t payload line is the same as the ones shown in Figure 6. For all mission opportunities, the Hybrid wet mass's sensitivity to the two power variables increases as the payload mass increases. The increase 


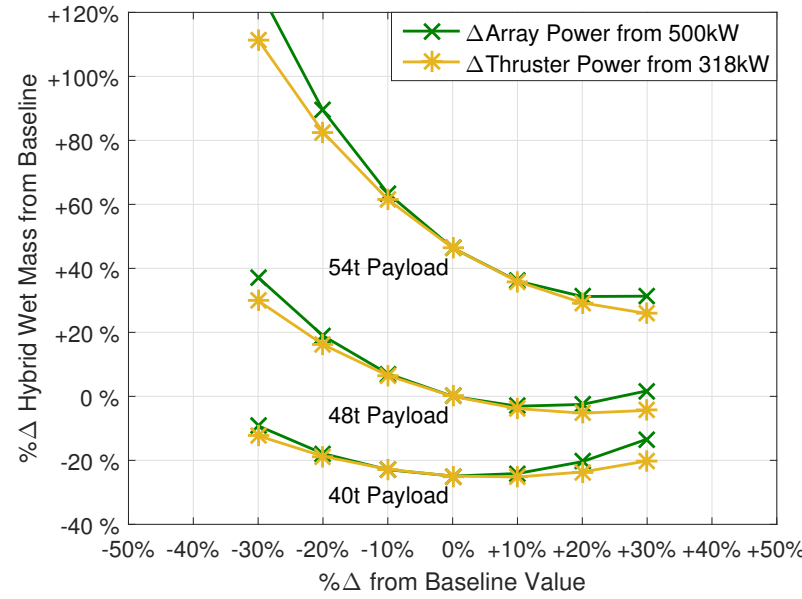

(a) 2033 Mission Opportunity

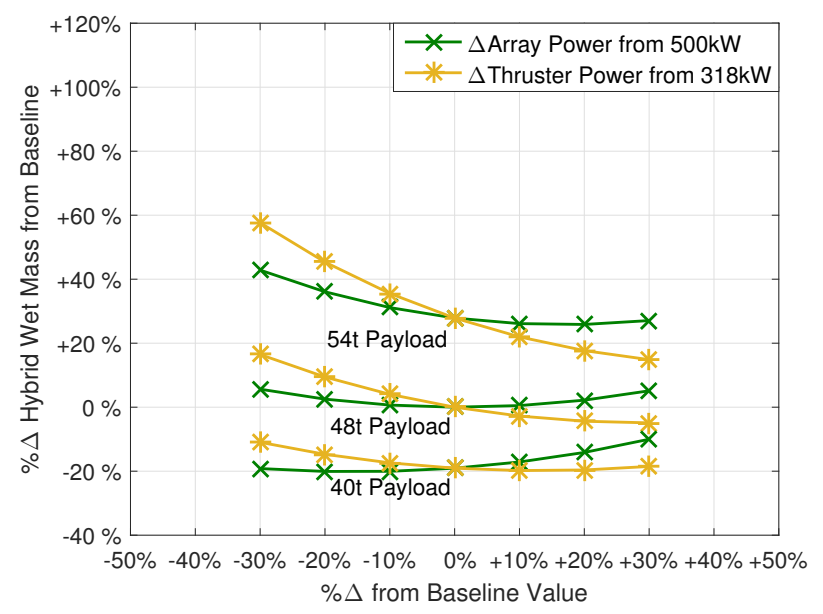

(c) 2041 Mission Opportunity

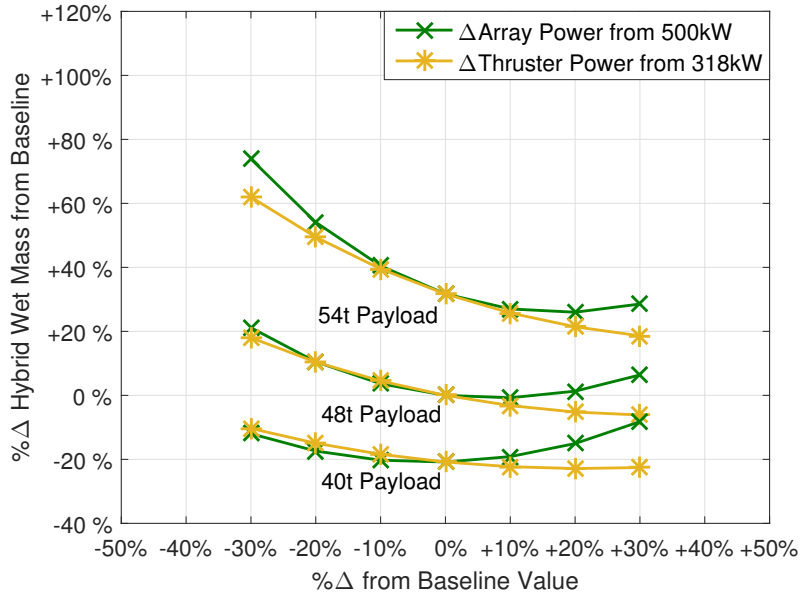

(b) 2037 Mission Opportunity

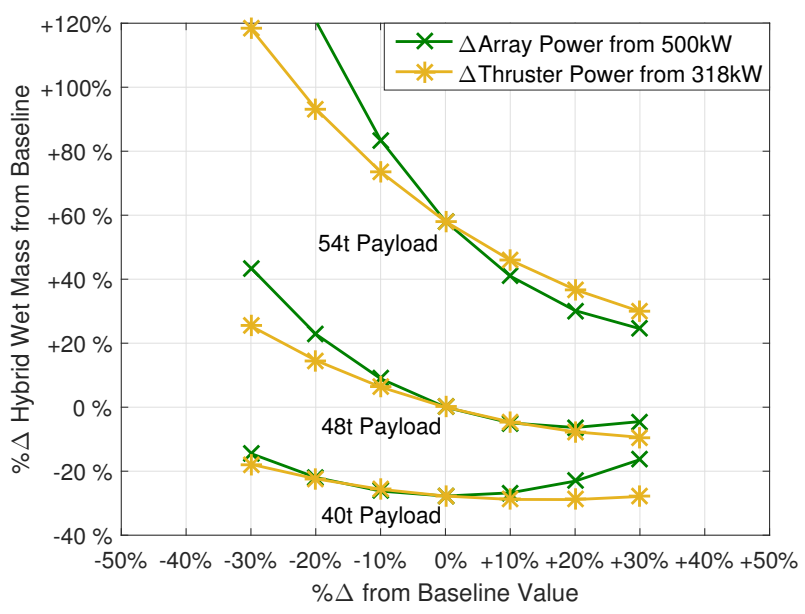

(d) 2045 Mission Opportunity

Figure 7: Sensitivity of the Hybrid Wet Mass as a Function of the Payload Mass, BOL Array Power, and SEP Thruster Power normalized to the Baseline Value

sensitivity is more profound for the 2033 and 2045 mission opportunities, as a $20-30 \%$ decrease in the power results in more than $100 \%$ increase in the Hybrid wet mass from the baseline for the 54 t payload case. Similar to the trend shown in Figure 5, the sensitivity to the power variables is reduced when the payload mass decreases or when the power variables are increased by 20-30\% from their baseline values. Both of these findings are critical in the design of the Hybrid transportation architectures. It highlights how the the increase in the payload mass has moved the Hybrid vehicle design space from a relatively robust portion of the performance curve to an area in which the Hybrid system mass is very sensitive to even minor changes to the SEP performance parameters.

\section{Summary}

The Mars Study Capability Team is continuing architectural trade analyses to define the capabilities and elements needed for a sustainable human presence on the surface of Mars. The Hybrid transportation system is being considered as the Deep Space Transport vehicle for the Mars campaign. Analysis and trades for the performance of the Hybrid system are ongoing, and as the architecture matures, it is inevitable that the payload mass and the performance of the propulsion system will change. It is desirable to understand how these changes will impact the in-space transportation system's mass and power requirements. This study serves to provide sensitivity analysis of the vehicle to both payload mass growth and SEP system 
performance parameters.

For this study, a parametric model of the Hybrid vehicle was developed from previous point designs to facilitate the sensitivity study. The results of the sensitivity analysis show that with the recent payload mass growth, the Hybrid vehicle is not operating at the optimal range of the current SEP system. The baseline SEP system parameters result in a Hybrid vehicle that is very sensitive to changes to those parameters from a mass perspective. At the current payload mass, an increase of 10-20\% of the SEP system power parameters will result in both reduction of the Hybrid system mass and improvement to the system's robustness to SEP system performance degradation. Additional increase to the SEP system performance parameters is required to increase the Hybrid system mass robustness if the payload mass is increased from the current baseline value.

The analyses in this study also show the difficulty in designing a reusable transportation system that can operate across multiple mission opportunities: the propulsive requirement for the SEP system and the chemical propulsion system can be significantly different. Optimal SEP thruster power and BOL array power for one mission opportunity can be wholly inadequate for a different opportunity. However, from a campaign planning and technology investment standpoint, it is infeasible to design a new vehicle that is optimal for each mission opportunity when the vehicle is being reused. Thus, it is vital to conduct these sensitivity analyses to understand the changes in the performance requirements across multiple opportunities and for varying payloads to determine the mass optimal vehicle design that is robust across the various mission requirements. Additional work is required to further refine the analyses presented in this study to include a more comprehensive design space and to capture the uncertainty in the vehicle sizing. The study also only considered the sensitivity of the mass of the system to the different performance parameters, when in reality these performance parameters may be dictated by other architecture level metrics such as cost, risk, and schedule. These metrics will need to be included in future studies to enable a Mars campaign that is truly robust and sustainable.

\section{References}

\footnotetext{
${ }^{1}$ Craig, D. A., Herrmann, N. B., and Troutman, P. A., "The Evolvable Mars Campaign - Study Status," IEEE Aerospace Conference, March 2015, 2015-8.0101.

${ }^{2}$ Goodliff, K., Troutman, P. A., Craig, D. A., and Herrmann, N. B., "Evolvable Mars Campaign 2016 Analysis Update," AIAA SPACE 2016 Conference and Exposition, 2016.

${ }^{3}$ Mars Architecture Steering Group and Drake, B. G., "Human Exploration of Mars Design Reference Architecture 5.0," Special Publication 2009-566, National Aeronautics and Space Administration, July 2009.

${ }^{4}$ Stanley, D. O., Cook, S., Connolly, J., Hamaker, J., Ivins, M., Peterson, W., Geffre, J., Cirillo, B., McClesky, C., Hanley, J., et al., "NASA's Exploration System Architecture Study," Technical Memorandum 2005-214062, National Aeronautics and Space Administration, November 2005.

${ }^{5}$ Olson, J., "Human Exploration Framework Team Phase I Closeout," National Aeronautics and Space Administration, September 2, 2010.

${ }^{6}$ Merrill, R. G., Strange, N., Qu, M., and Hatten, N., "Mars Conjunction Crewed Missions with a Reusable Hybrid Architecture," IEEE Aerospace Conference, March 2015, 2015-8.0104.

${ }^{7}$ Hofer, R. and Gallimore, A., "High-Specific Impulse Hall Thrusters, Part 1: Influence of Current Density and Magnetic Field," Journal of Propulsion and Power, Vol. 22, No. 4, 2006, pp. 721-731.

${ }^{8}$ Hofer, R. and Gallimore, A., "High-Specific Impulse Hall Thrusters, Part 2: Efficiency Analysis," Journal of Propulsion and Power, Vol. 22, No. 4, 2006, pp. 732-740.

${ }^{9}$ Chai, P. R., Merrill, R. G., and Qu, M., "Mars Hybrid Propulsion System Trajectory Analysis Part I: Crew Missions," AIAA SPACE 2015 Conference and Exposition, Pasadena, CA, August 2015, AIAA 2015-4443.

${ }^{10}$ Chai, P. R., Merrill, R. G., and Qu, M., "Mars Hybrid Propulsion System Trajectory Analysis Part II: Cargo Missions," AIAA SPACE 2015 Conference and Exposition, Pasadena, CA, August 2015, AIAA 2015-4444.

${ }^{11}$ Chai, P. R., Qu, M., and Merrill, R. G., "End-to-End Trajectory for Conjunction Class Mars Missions using Hybrid SolarElectric/Chemical Transportation System," American Astronautical Society's 26th Space Flight Mechanics Meeting, 2016, AAS $16-255$.

${ }^{12} \mathrm{Qu}$, M., Merrill, R. G., Chai, P. R., and Komar, D. R., "Trajectory Designs for a Mars Hybrid Transportation Architecture," 2015 AAS/AIAA Astrydynamics Specialist Conference, August 2015, AAS 15-552.

${ }^{13}$ Merrill, R. G., Chai, P. R., and Min, "An Integrated Hybrid Transportation Architecture for Human Mars Expeditions," AIAA SPACE 2015 Conference and Exposition, Pasadena, CA, 2015, AIAA 2015-4442.

${ }^{14}$ McGuire, M., Oleson, S., Babula, M., and Sarver-Verhey, T., "Concurrent Mission and System Design at NASA Glenn Research Center: The origins of the COMPASS Team," AIAA SPACE 2011 Conference 83 Exposition, Long Beach, CA, September 2011, AIAA 2011-7240.

${ }^{15}$ Aerojet-Ricketdyne, Inc., "Bipropellant Rocket Engine Data Sheets," Aeroject-Rocketdyne Inc., Space Propulsion Systems, Space and Launch, Capabilities, https://www.rocket.com/files/aerojet/documents/Capabilities/PDFs/Bipropellant $\% 20$ Data\%20Sheets.pdf [Accessed: July 1, 2015].
} 
${ }^{16}$ Sims, J. A., Finlayson, P. A., Rinderle, E. A., Vavrina, M. A., and Kowalkowski, T. D., "Implementation of a LowThrust Trajectory Optimization Algorithm for Preliminary Design," AIAA/AAS Astrodynamics Specialist Conference and Exhibit, 2006, AIAA 2006-6746.

${ }^{17}$ Ewert, M. K., Broyan, J. L., Semones, E. J., Goodliff, K. E., Chai, P. R., Singleterry, R. C., Abston, L., Clowdsley, M. S., Wittkopp, C. J., and Vitullo, N. A., "Comparing Trash Disposal to Use as Radiation Sheilding for a Mars Transit Vehicle," 47th International Conference on Environmental Systems, 2017, ICES-2017-178. 